\title{
Coronary Occlusion Detection with 4D Optical Flow Based Strain Estimation on 4D Ultrasound
}

\author{
Qi Duan ${ }^{1,2}$, Elsa D. Angelini ${ }^{3}$, Auranuch Lorsakul ${ }^{1}$, Shunichi Homma ${ }^{1}$, and \\ Andrew F. Laine ${ }^{1}$ \\ ${ }^{1}$ Columbia University, New York, NY, USA \\ $\{$ qd2002, al2776, sh23, laine\}@columbia.edu \\ ${ }^{2}$ Center for Biomedical Imaging, NYU School of Medicine, New York, NY, USA \\ Qi.Duan@nyumc.org \\ ${ }^{3}$ Institut Telecom, Telecom-ParisTech, CNRS LTCI, Paris, France \\ elsa.angelini@telecom-paristech.fr
}

\begin{abstract}
Real-time three-dimensional echocardiography (RT3DE) offers an efficient way to obtain complete 3D images of the heart over an entire cardiac cycle in just a few seconds. The complex $3 \mathrm{D}$ wall motion and temporal information contained in these 4D data sequences has the potential to enhance and supplement other imaging modalities for clinical diagnoses based on cardiac motion analysis. In our previous work, a 4D optical flow based method was developed to estimate dynamic cardiac metrics, including strains anddisplacements, from 4D ultrasound. In this study, in order to evaluate the ability of our method in detecting ischemic regions, coronary artery occlusion experiments at various locations were performed on five dogs. 4D ultrasound data acquired during these experiments were analyzed with our proposed method. Ischemia regions predicted by the outcome of strain measurements were compared to predictions from cardiac physiology with strong agreement. This is the first direct validation study of our image analysis method for clinical diagnoses and outcome.
\end{abstract}

\section{Introduction}

Motion estimation in echocardiography has been and continues to be an active area of research. Doppler ultrasound has become widespread for imaging flow and quantifying blood and tissue velocities. However, this method is limited by angular dependency, i.e., it can only quantify the axial component of motion along the transmitted acoustic wave. Speckle tracking methods [1-4] overcome this limitation by directly tracking the backscattered echoes produced by the ultrasonic scatterers in blood and tissues. The tracking can be performed either in the image domain [2-4] or directly in the radio-frequency (RF) domain [1]. Although in theory this can be done on 1D, 2D, and 3D images, clinical applications are limited to $1 \mathrm{D}$ and 2D modalities. The ability of speckle tracking to estimate motion and strain has been validated against MR tagging in recent studies [5].

Development of real-time 3D (RT3D) echocardiography started in the late 1990s [6] based on matrix phased array transducers. Recently, a new generation of RT3D transducers was introduced by Philips Medical Systems (Andover, MA) in the SONOS 7500 , followed by the iE33 model, which can acquire a fully sampled cardiac volume in four cardiac cycles and capture complex 4D cardiac motion well. However, full exploitation of three-dimensional ultrasound data for qualitative and quantitative evaluation of cardiac function remains sub-optimal due to the lack of proper 4D analysis tools. Manual tracing of myocardial borders is a tedious task that requires the 
intervention of an expert cardiologist familiar with the ultrasound machine and an interactive drawing interface. For this reason, myocardial motion is commonly assessed via visual inspection of B-scan images. Moreover, to this date, there is no commercial real-time 3D ultrasound machine or software that can provide direct quantitative motion and strain measurements analogous to tissue Doppler or strain imaging, which has limited the application of RT3D echocardiography to anatomical measurements and visual inspection of cardiac motion.

For this reason, several image processing-based motion and strain estimations using 3D ultrasound have been proposed. Meunier [3] showed the performance of 3D speckle tracking using simulated ultrasound images. Our group [7, 8] has shown that using a correlation-based optical flow method, myocardial motion fields can be estimated, from which directional displacements and strains can be computed. In order to evaluate the values in clinical diagnosis, in this study, ischemia regions predicted by our method were directly compared to cardiac physiology prediction during controlled coronary occlusion experiments.

\section{Method}

\subsection{Correlation-based Optical Flow}

Optical flow (OF) tracking refers to the computation of the displacement field of objects in an image, based on the assumption that the intensity of the object remains constant. In this context, motion of the object is characterized by a flow of pixels with constant intensity. The assumption of intensity conservation is typically unrealistic for natural movies and medical imaging applications, motivating the argument that $\mathrm{OF}$ can only provide qualitative estimation of object motions. There are two global families of OF computation techniques: (1) Differential techniques [9-11] that compute velocity from spatio-temporal derivatives of pixel intensities; (2) Regionbased matching techniques $[12,13]$, which compute the OF via identification of local displacements that provide optimal correlation of two consecutive image frames. Compared to differential OF approaches, region-based methods using correlation measures are less sensitive to noisy conditions and fast motion [14] but assume that displacements in small neighborhoods are similar. In three-dimensional ultrasound, this later approach appeared more appropriate and was selected for this study. Given two data sets from consecutive time frames: $(I(\mathbf{x}, t), I(\mathbf{x}, t+\Delta t))$, the displacement vector $\Delta \mathbf{x}$ for each pixel in a small neighborhoods $\Omega$ around a pixel $x$ is estimated via maximization of the cross-correlation coefficient defined as:

$$
r=\frac{\sum_{\mathbf{x} \in \Omega}(I(\mathbf{x}, t) * I(\mathbf{x}+\Delta \mathbf{x}, t+\Delta t))}{\sqrt{\sum_{\mathbf{x} \in \Omega} I^{2}(\mathbf{x}, t) \sum_{\mathbf{x} \in \Omega} I^{2}(\mathbf{x}+\Delta \mathbf{x}, t+\Delta t)}}
$$

In this study, correlation-based OF was applied to estimate the displacement of selected voxels between two consecutive ultrasound volumes in the cardiac cycle. The search window $\Omega$ was centered about every $(5 \times 5 \times 5)$ pixel volume and was set to size $(7 \times 7 \times 7)$. 


\subsection{Cardiac Dynamic Metrics}

A flowchart of the computational framework is provided in Figure 1 below. Three coordinate systems are involved in the computational framework (Figure 2): pixel coordinates $(\mathrm{i}, \mathrm{j}, \mathrm{k})$, Cartesian coordinates $(\mathrm{x}, \mathrm{y}, \mathrm{z})$, and cylindrical coordinates ( $\mathrm{r}, \theta$, $z)$. The OF estimation was performed in pixel coordinates. For the computation of dynamic information, displacements in pixel coordinates were converted into Cartesian coordinates and centered inside the ventricular cavity so that the z-axis was aligned with the long axis of left ventricle. This coordinate transform is performed via the rigid transformation:

$$
\left[\begin{array}{l}
x \\
y \\
z
\end{array}\right]=R\left[\begin{array}{l}
i \\
j \\
k
\end{array}\right]+T=\left[\begin{array}{lll}
r_{11} & r_{12} & r_{13} \\
r_{21} & r_{22} & r_{23} \\
r_{31} & r_{32} & r_{33}
\end{array}\right]\left[\begin{array}{l}
i \\
j \\
k
\end{array}\right]+\left[\begin{array}{l}
-O_{i} \\
-O_{j} \\
-O_{k}
\end{array}\right],
$$

where $R$ is a rotation matrix, $T$ is a translation vector, equal to the negative pixel coordinates of the origin $O$ of the Cartesian coordinate system. The ventricular axis was defined as the axis connecting the center of the mitral orifice and the endocardial apex. This axis has a very stable position during the whole cardiac cycle [15]. Based on the Cartesian coordinate system, a corresponding cylindrical coordinate system is established with the $r$ - $\theta$ plane corresponding to the $x-y$ plane and with the $x$-axis used as the reference for $\theta$.

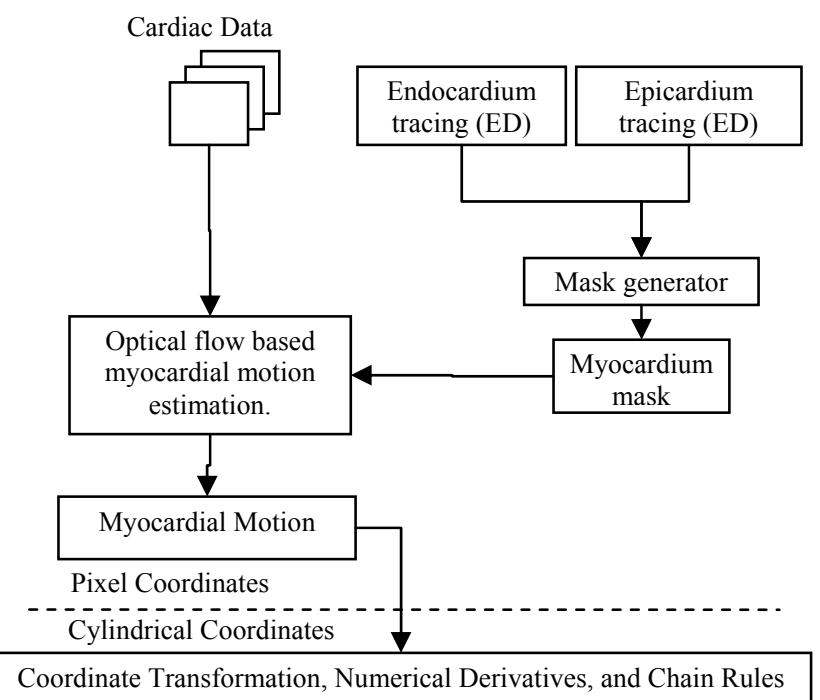

Figure 1: Flow chart of computational framework for processing of 4D echocardiography data for measures of strain. 


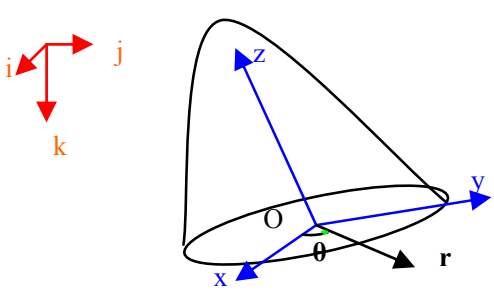

Figure 2. Coordinate systems for data acquisition and computation.

Endocardial and epicardial tracing were provided by cardiologists at the enddiastole, i.e. the $1^{\text {st }}$ frame of the $4 \mathrm{D}$ ultrasound sequence, as an initialization. Based on that, myocardium voxels were segmented and automatically tracked via correlationbased optical flow algorithm through the whole cardiac cycle. Myocardial motion field $\left(u_{x}, u_{y}, u_{z}\right)$ for each myocardial voxel provided by optical flow was then used to compute dynamic cardiac metrics, including:

- Displacement low magnitude $|u|(\mathrm{mm})$

- $\quad$ Radial displacement $u_{r}(\mathrm{~mm})$

- Circumferential displacement $u_{\theta}(\mathrm{mm})$

- Strain Tensor $E=\left(\nabla u+\nabla u^{T}+\nabla u^{T} \nabla u\right) / 2$, where the major diagonal values of $E$ contain thickening, circumferential strain, and longitudinal strain

- Twist $\partial u_{\theta} / \partial z$ and cardiac torsion.

Gradient values were computed directly in pixel coordinates and converted into cylindrical coordinate system via chain rule. Derivatives in pixel coordinates were approximated by central difference operators.

Since in clinical applications, temporal profiles of the segmental average values for displacements and strains are more valuable than ED-to-ES values, we modified our software so that it could compute temporal profiles of these cardiac dynamic metrics in segmental average fashion, according to the 16-segment model of the left ventricular myocardium according to the American Society of Echocardiography [16].

\subsection{Controlled Occlusion Experiments}

To validate the ability of the proposed framework in predicting ischemia regions, several controlled occlusions during open heart canine experiments were performed under a protocol approved by Columbia University's Institutional Animal Care and Use Committee. In each experiment, 4 different occlusions, including distal and proximal LAD occlusion and distal and proximal LCx occlusions were performed. 4D ultrasound data was acquired during the experiment both for baseline and 2 minutes after occlusions with the iE33 ultrasound machine (Philips, Andover, MA). The same experiment protocol was repeated for five different dogs on five different days. 


\section{Results}

\subsection{Temporal Curves}

Results of radial displacements and radial strain (thickening) on the baseline scan and after proximal LAD occlusion are shown in Figure 3 and Figure 4 respectively. Compared to the results of the baseline scan, radial displacement correctly indicated the outward motion of the anterior section and reduced motion for antero-septal and septal sections of the heart. Overall motion magnitudes were lower for the other three sections. The radial strain analysis results were even more interesting as : (1) negative radial strain values were observed for the anterior section throughout the whole cardiac cycle, (2) values for antero-septal segments also dropped, (3) whereas the values of lateral and posterior sections were relatively stable.

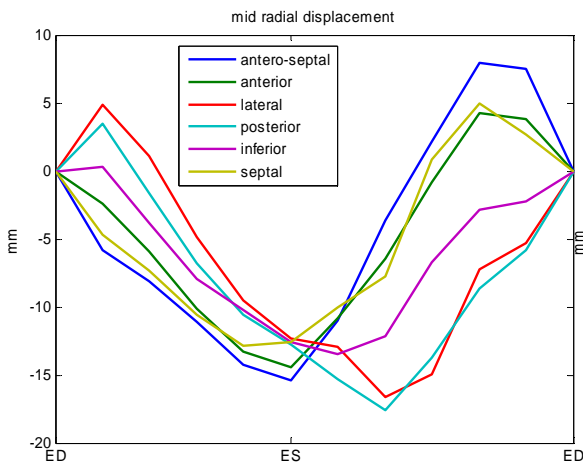

(a)

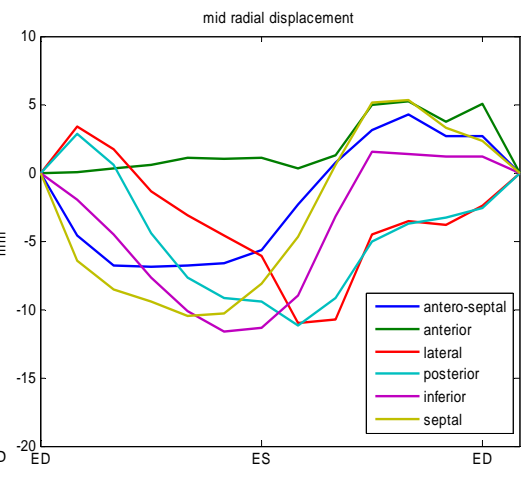

(b)

Figure 3: Sample radial displacement estimation from 4D ultrasound analysis on (a) baseline and (b) ischemic scan in canine with proximal LAD occlusion.

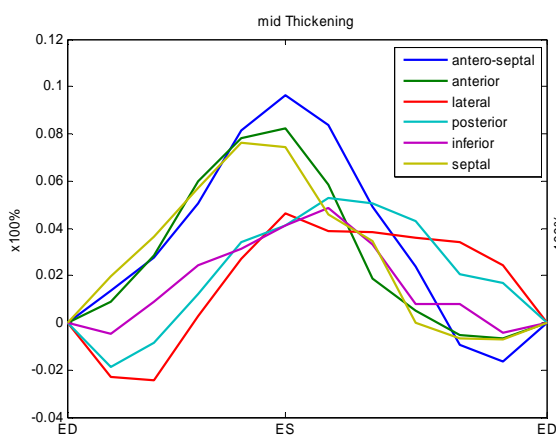

(a)

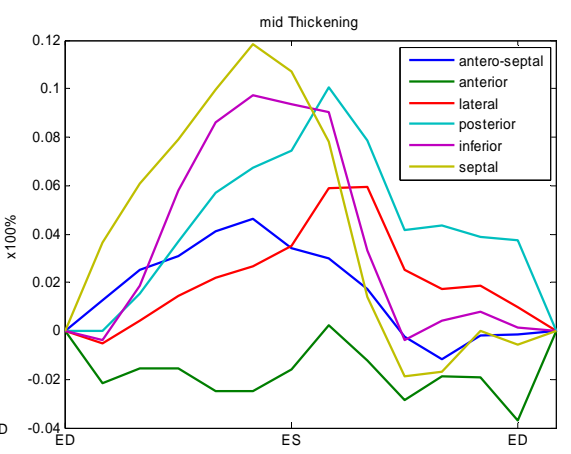

(b)

Figure 4: Sample radial strain (thickening) estimation from 4D ultrasound analysis on (a) baseline and (b) ischemic scans in canine with proximal LAD occlusion. 
Similar results can also be found from the circumferential strain values in Figure 5: Under LAD occlusion, values on the anterior segment changed from normal values (negative) to abnormal (positive); values of antero-septal segments also dropped in magnitude, whereas values of lateral and posterior sections remained relatively stable.

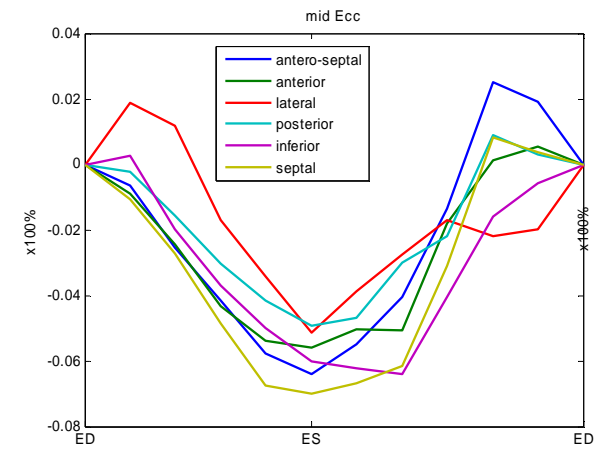

(a)

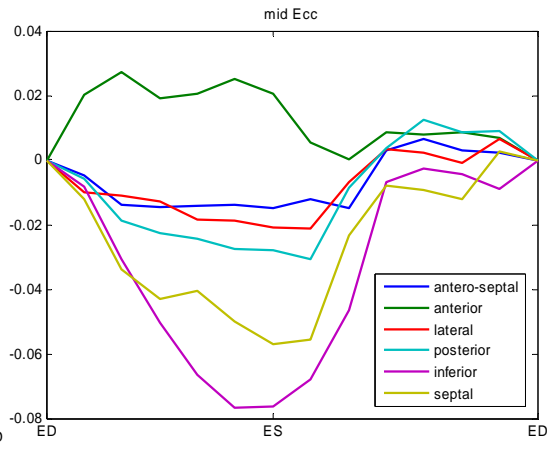

(b)

Figure 5: Sample circumferential strain estimation from 4D ultrasound analysis on (a) baseline and (b) ischemic scans in canine with proximal LAD occlusion.

\subsection{ED-ES 3D results}

We also compared our findings based on optical flow strain on canine heart experimental data having a proximal LAD occlusion with predicted measures from a physiological finite element model [15] under the same occlusion status. Sample results are shown in Figure 6. The abnormal region found by our proposed method of 4D optical flow (shown in red in Figure 6b) were very similar to the model prediction (shown in Figure 6a). Besides radial displacement, other metrics, such as wall thickening and cardiac torsion, have yielded similar results of agreement.

3D visualizations made with CardioViz3D software [17] of selected dog studies data are shown in Figure 7. The studies are the baseline case and the proximal LAD occlusion case from the same dog side by side. The colors displayed on the 3D meshes are mapped to strain values rescaled between -1 and 1. From the results, the normal case on anterior and posterior views in Figure $7 \mathrm{a}$ and $7 \mathrm{~b}$ shows mostly a green color mapping, while in the proximal case regions of blue color are shown in specific areas. In this example, we can therefore easily localize the abnormal area on the posterior view in Figure 7d. This result was compared to standard views for twodimensional echocardiography, and can indicate abnormal regions due to ischemia. These results also coincide with cardiac physiology findings for each case. 
base

ant

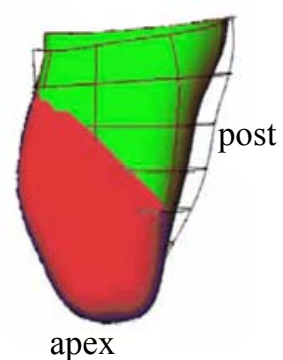

(a)
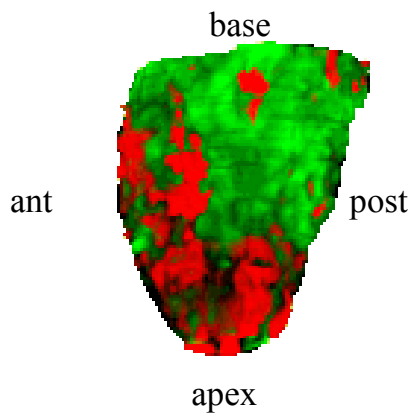

apex

Figure 6: (a) Anterior view of the finite element model with a large simulated LAD occlusion. Wire frame represents the endocardial surface at end diastole, solid surface the endocardial surface at end systole, and the red portion corresponds to the deactivated (ischemic) region. (b) $3 \mathrm{D}$ visualization of ED-to-ES radial displacement metric of a canine heart experiment with proximal LAD occlusion, which is very similar to predicted values of the physiological model.

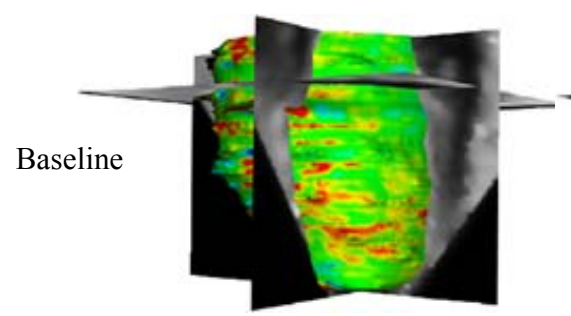

(a)

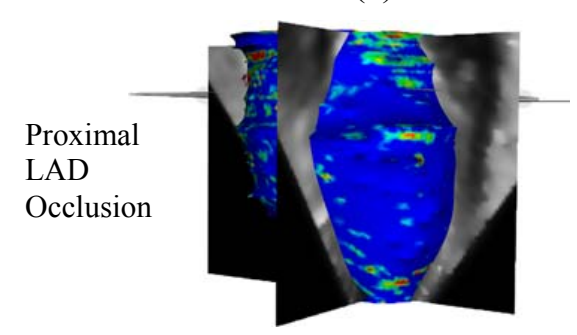

(c)

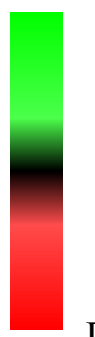

Dyskinetic

Normal

Akinetic

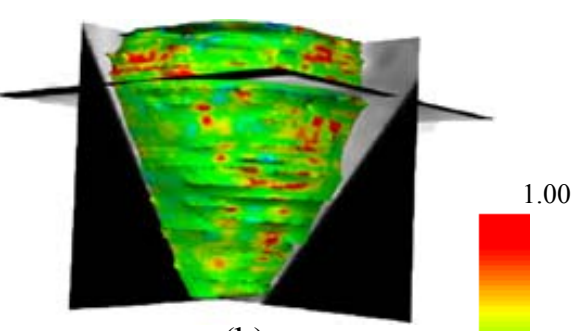

(b)

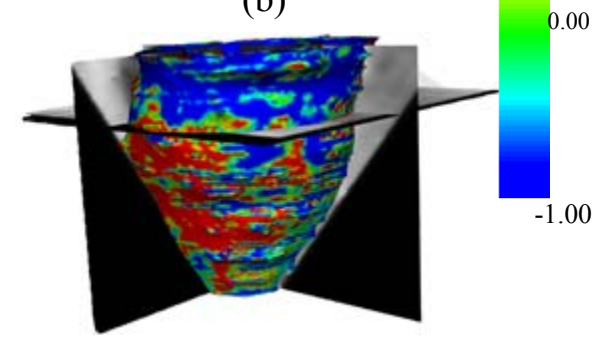

(d)

Anterior View

Posterior View

Figure 7. 3D visualization of dog (canine) cardiac study (a) Anterior view of the baseline case (b) Posterior view of the baseline case (c) Anterior view of the proximal LAD occlusion (d) Posterior view of the proximal LAD occlusion. 


\section{Conclusion}

A correlation-based optical flow (OF) method was applied to myocardial tracking on 4D ultrasound data. Based on the estimated motion fields, several dynamic cardiac metrics, including displacements and strain, were derived. The proposed method was applied to 4D ultrasound data acquired via controlled coronary artery occlusion. Ischemic regions predicted by temporal profiles of strain as well as ED-ES strain maps were aligned with predictions from a cardiac physiological model, simulated with the same occlusion position and extent. In the future, a finite-element based fitting could further improve both the accuracy and robustness of our proposed computational framework. The ability to derive strain measurement from real-time 3D ultrasound is of great clinical and practical importance. Thus, our method could provide a novel diagnostic tool that would allow researchers and clinicians to use 4D strain estimation based on echocardiography.

\section{Acknowledgements}

The authors also would like to thank Dr. Holmes (Department of Biomedical Engineering, University of Virginia, USA) for performing the canine experiments.

\section{References}

1. M. A. Lubinski, S. Y. Emelianov, and M. O'Donnell, "Speckle Tracking Methods for Ultrasonic Elasticity Imaging Using Short-Time Correlation," IEEE Transactions on Ultrasonics, Ferroelectrics, and Frequency Control, vol. 46, pp. 82-96, 1999.

2. K. Kaluzynski, X. Chen, S. Y. Emelianov, A. R. Skovoroda, and M. O'Donnell, "Strain Rate Imaging Using Two-Dimensional Speckle Tracking," IEEE Transactions on Ultrasonics, Ferroelectrics, and Frequency Control, vol. 48, 2001.

3. J. Meunier, "Tissue motion assessment from 3D echographic speckle tracking," Physics in Medicine and biology, vol. 43, pp. 1241-1254, 1998.

4. L. N. Bohs, B. J. Geiman, M. E. Anderson, S. C. Gebhart, and G. E. Trahey, "Speckle tracking for multidimensional flow estimation," Ultrasonics, vol. 38, pp. 369-375, 2000.

5. Y. Notomi, P. Lysyansky, R. M. Setser, T. Shiota, Z. B. Popovic, M. G. Martin-Miklovic, J. A. Weaver, S. J. Oryszak, N. L. Greenberg, R. D. White, and J. D. Thomas, "Measurement of Ventricular Torsion by Two-Dimensional Ultrasound Speckle Tracking Imaging," Journal of the American College of Cardiology, vol. 45, pp. 2034-2041, 2005.

6. O. T. V. Ramm and S. W. Smith, "Real time volumetric ultrasound imaging system," Journal of Digital Imaging, vol. 3, pp. 261-266, 1990.

7. Q. Duan, E. Angelini, O. Gerard, S. Homma, and A. Laine, "Comparing optical-flow based methods for quantification of myocardial deformations on RT3D ultrasound," presented at IEEE International Symposium on Biomedical Imaging (ISBI), 2006.

8. Q. Duan, E. Angelini, S. L. Herz, C. M. Ingrassia, O. Gerard, K. D. Costa, J. W. Holmes, S. Homma, and A. Laine, "Dynamic Cardiac Information From Optical Flow Using Four Dimensional Ultrasound," presented at 27th Annual International Conference IEEE Engineering in Medicine and Biology Society (EMBS), Shanghai, China, 2005.

9. B. D. Lucas and T. Kanade, "An iterative image registration technique with an application to stereo vision," presented at International Joint Conference on Artificial Intelligence (IJCAI), 1981.

10. B. K. P. Horn and B. G. Schunck, "Determining optical flow," Artificial Intelligence, vol. 17, 1981.

11. H. Nagel, "Displacement vectors derived from second-order intensity variations in image sequences," Computer Vision Graphics Image Processing, vol. 21, pp. 85-117, 1983.

12. P. Anandan, "A computational framework and an algorithm for the measurement of

visual motion," International Journal of Computer Vision, vol. 2, pp. 283-310, 1989.

13. A. Singh, "An estimation-theoretic framework for image-flow computation," presented at International Conference on Computer Vision, 1990.

14. J. L. Barron, D. Fleet, and S. Beauchemin, "Performance of optical flow techniques," Int. Journal of Computer Vision, vol. 12, pp. 43-77, 1994. 
15. S. Herz, C. Ingrassia, S. Homma, K. Costa, and J. Holmes, "Parameterization of left ventricular wall motion for detection of regional ischemia.," Annals of Biomedical Engineering, vol. 33, pp. 912-919, 2005.

16. M. Cerqueira, N. Weissman, V. Dilsizian, A. Jacobs, S. Kaul, W. Laskey, D. Pennell, J. Rumberger, T. Ryan, and M. Verani, "Standardized myocardial segmentation and nomenclature for tomographic imaging of the heart," Circulation, vol. 105, pp. 539-542, 2002.

17. N. Toussaint, T. Mansi, H. Delingette, N. Ayache, and M. Sermesant, "An Integrated Platform for Dynamic Cardiac Simulation and Image Processing: Application to Personalised Tetralogy of Fallot Simulation," presented at Proc. Eurographics Workshop on Visual Computing for Biomedicine (VCBM), Delft, The Netherlands, 2008. 